\title{
Phase Transition and Mechanical Stability of InN Compound
}

\author{
A. Khaldi ${ }^{a, b, *}$, N. BouArissa ${ }^{c, d}$ AND L. TABOUROT ${ }^{e}$ \\ ${ }^{a}$ Laboratory of Materials and Electronic Systems (LMSE), University of Mohamed El Bachir El Ibrahimi, \\ 34000, Bordj Bou Arreridj, Algeria \\ ${ }^{b}$ Department of Civil Engineering, Faculty of Science and Technology, University of Bordj Bou Arreridj, \\ 34000, Algeria \\ ${ }^{c}$ Laboratory of Materials Physics and Its Applications, University of M'sila, 28000 M'sila, Algeria \\ ${ }^{d}$ Physics Department, Faculty of Science, University of M'sila, 28000 M'sila, Algeria \\ ${ }^{e}$ Université Savoie Mont Blanc, SYMME, F-74000 Annecy, France
}

\begin{abstract}
$A b$ initio calculations of structural phase transition and mechanical stability of zinc-blende InN compound have been conducted in this work using the pseudopotential plane-wave method within the generalized gradient approximation. The first transition pressure of the material in question has been determined and compared to the data available in the literature. In addition, the mechanical stability criteria have been checked which indicate that zinc-blende $\mathrm{InN}$ is mechanically stable for all pressures of interest up to the transition pressure.
\end{abstract}

DOI: 10.12693/APhysPolA.137.480

PACS/topics: phase transition, elastic properties, InN

\section{Introduction}

The group-III-nitrides are of potential interest as optical devices for the optoelectronic technology. This is because of their promising potential to be used for hightemperature, high-frequency, and high-power electronics. These materials are characterized by their wide band gaps and strong bond strength. This makes them being increasingly used for visible light emitting diodes and laser diodes [1-13]. Indium nitride ( $\mathrm{InN})$ is an actively investigated semiconductor that has technological applications in optoelectronic devices emitting in the blue light and green ultraviolet spectral regions [14-20]. InN adopts preferentially the hexagonal wurtzite structure, nevertheless, the cubic zinc-blende form has been reported for this material $[2,21-23]$. This form has technological advantages, such as easier doping, easier cleaning (for laser facets), and easier contacting.

The study of the effect of high-pressure on the fundamental properties of semiconductors has been increased during the recent years [24-32]. This is mainly due to the development of the diamond anvil cell and a rapid increase in computer power. Although some papers investigated the mechanical properties of $\operatorname{InN}[14-17,33]$, only a few of them dealt with the effect of pressure on these properties. This has prompted us to take up such a study for InN in the zinc-blende structure. Our study is focused on the transition phase and mechanical stability of InN under high-pressure. The calculations are performed using ab initio calculations based on the density functional theory (DFT) within the generalized gradient approximation (GGA).

*corresponding author; e-mail: a.khaldi@univ-bba.dz

\section{Computational method}

The calculations are perfumed using a pseudopotential plane-wave method as implemented in the CASTEP code [34]. The Vanderbilt-type ultra-soft pseudopotentials [35] are used in order to describe the interaction between valence electrons and ion cores of In and N. The GGA of Perdew et al. for solids [36] is used to treat the exchange-correlation energy.

The plane wave cut off energy is taken to be $400 \mathrm{eV}$. The Brillouin zone integrations are carried out using Monkhorst and Pack $k$-point meshes [37], with a $12 \times 12 \times 12$ special $k$ points mesh.

The finite strain-stress method [38] is used for determining the single-crystal elastic coefficients $\left(C_{i j}\right)$. By deriving $C_{i j}$, the polycrystalline elastic constants are calculated using the Voigt-Reuss-Hill approximations [39-44].

\section{Results and discussion}

Thermodynamically speaking, the lowest Gibbs free energy, given by the relation

$$
G=U+P V-T S,
$$

corresponds to the stability of any given structure. In Eq. (1), $U, P, V, T$, and $S$ are total internal energy, pressure, volume, temperature, and entropy, respectively. By optimizing the cell parameters and atomic positions for zinc-blende $(B 3)$ and $\mathrm{NaCl}(B 1)$ phases at $T=0 \mathrm{~K}$, one can obtain the pressure-induced structural transition. For that purpose and given the fact that $G$ becomes equal to the enthalpy $H$ at $T=0 \mathrm{~K}$, we have studied the variation of $H$ as a function of pressure for both phases being considered here. Our results are displayed in Fig. 1. Note that the two curves representing $H$ versus pressure for $B 3$ and $B 1$ phases cross at a pressure of $8.35 \mathrm{GPa}$. This suggests that the InN zinc-blende phase transforms to 


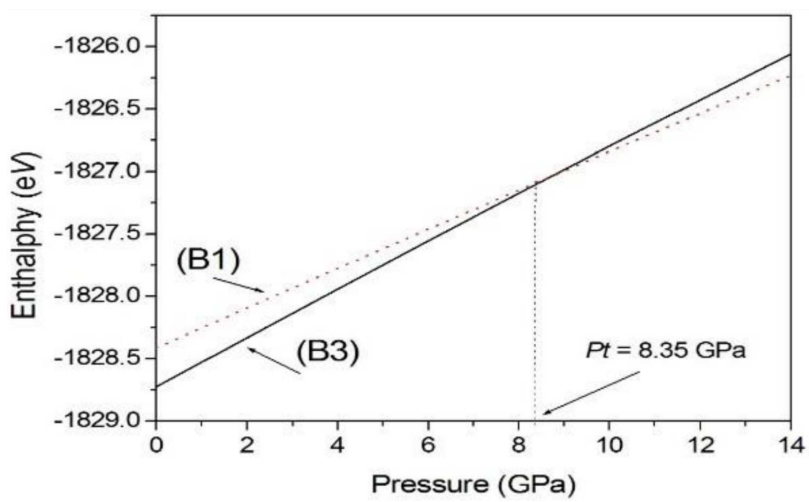

Fig. 1. Enthalpy versus pressure for zinc-blende (B3) and $\mathrm{NaCl}(B 1)$ phases of $\mathrm{InN}$.

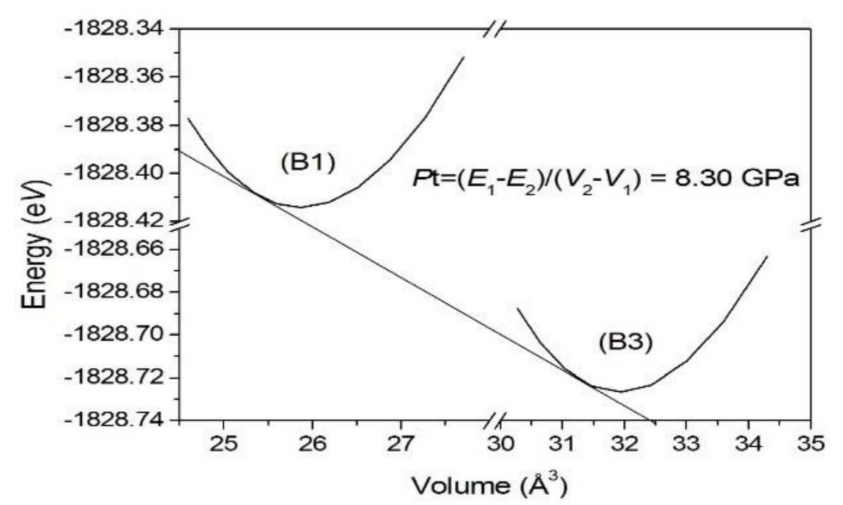

Fig. 2. Total energy versus volume for zinc-blende $(B 3)$ and $\mathrm{NaCl}(B 1)$ phases of $\mathrm{InN}$.

the $\mathrm{NaCl}$ phase at a transition pressure $P_{t}$ of $8.35 \mathrm{GPa}$. Beyond $P_{t}$, the $\mathrm{NaCl}$ phase becomes more stable than the zinc-blende phase.

The value of $P_{t}$ can also be obtained from the curves of total energies and relative volumes in $B 3$ and $B 1$ phases. In this method, $P_{t}$ is the gradient of common tangent given by [45]:

$$
P_{t}=-\frac{\Delta E}{\Delta V}=\frac{E_{1}-E_{2}}{V_{2}-V_{1}} .
$$

In the current contribution, the total energies versus relative volumes for both $\mathrm{InN}$ phases of interest are illustrated in Fig. 2. Our findings show that $P_{t}=8.30 \mathrm{GPa}$. This value is very close to the one we obtained via enthalpy. As compared to transition pressures reported in the literature for zinc-blende $\mathrm{InN}$, our findings seem to be smaller than those lying in the range 12.1-23.0 GPa, as suggested by Adachi [46].

The elastic constants of solid materials are important parameters that are critical for device fabrications and various fundamental properties of the materials are related to them [47-50]. In this work, the elastic constants of zinc-blende InN have been calculated. Our results yielded values of 168,113 , and $83 \mathrm{GPa}$ for $C_{11}, C_{12}$,

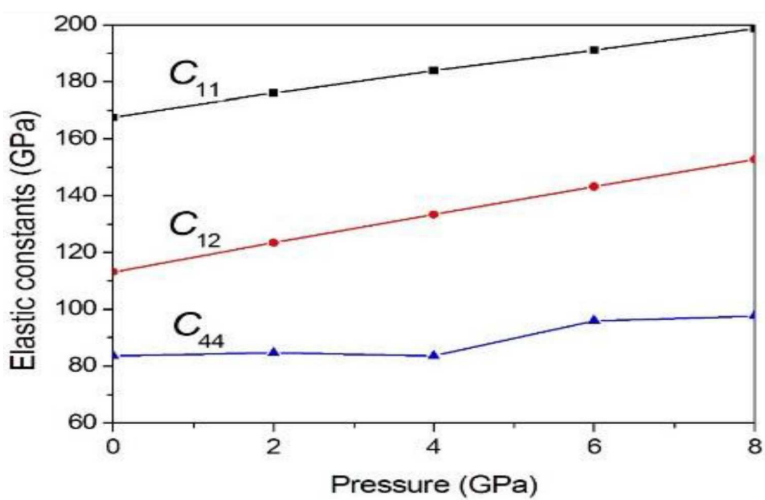

Fig. 3. Elastic constants versus pressure for zincblende (B3) InN.

and $C_{44}$, respectively. These values are respectively in reasonably good accord with those of 187, 125, and 86 GPa reported by Wright [17] using DFT calculations. The pressure dependence of these elastic constants is shown in Fig. 3. Note that all elastic constants of interest increase with enhancing pressure. The behavior appears to be linear for $C_{11}$ and $C_{12}$, but non-linear for $C_{44}$.

In order to check the mechanical stability of the material in question under pressure, we have used the traditional mechanical stability criteria for cubic crystals under pressure [31, 43],

$K=\frac{1}{3}\left(C_{11}+2 C_{12}+P\right)>0$,

$G=\frac{1}{2}\left(C_{11}-C_{12}-2 P\right)>0, \quad G^{\prime}=C_{44}-P>0$.

Our findings showed that all the criteria are fulfilled for each given pressure of interest. This indicates that zinc-blende $\mathrm{InN}$ is mechanically stable for all pressure up to $P_{t}$.

\section{Conclusion}

In summary, based on ab initio pseudopotential planewave method within the GGA, the pressure dependence of structural and elastic properties of $\mathrm{InN}$ were investigated. Our results showed that InN transforms from $B 3$ to $B 1$ at a pressure of about $P_{t}=8 \mathrm{GPa}$. The material of interest is found to be mechanically stable up to a pressure of $P_{t}$.

\section{References}

[1] J.W. Orton, C.T. Foxon, Rep. Prog. Phys. 61, 1 (1998) and references therein.

[2] S.C. Jain, M. Willander, J. Narayan, R. Van Overstraeten, J. Appl. Phys. 87, 965 (2000).

[3] N. Bouarissa, Phys. Status Solidi B 231, 391 (2002).

[4] I. Vurgaftman, J.R. Meyer, J. Appl. Phys. 94, 3675 (2003) and references therein.

[5] S. Saib, N. Bouarissa, J. Phys. Chem. Solids 67, 1888 (2006). 
[6] S. Strite, H. Morkoç, J. Vac. Sci. Technol. B10, 1237 (1992)

[7] H. Morkoç, S.N. Mohammad, Science 267, 51 (1995).

[8] N. Bouarissa, Philos. Mag. B 80, 1743 (2000).

[9] K. Kassali, N. Bouarissa, Microelectron. Eng. $\mathbf{5 4}$ 277 (2000).

[10] M. Asim, Emerg. Mater. Res. 8, 320 (2019).

[11] S. Naim, Z. Arbër, H. Nazmi, E. Njomza, Emerg. Mater. Res. 9, 1 (2020).

[12] R.B. Patel, T. Chou, Z. Iqbal, Emerg. Mater. Res. 3, 115 (2014)

[13] S.N. Bahadur, A. Sonal, Emerg. Mater. Res. 5, 5 (2016).

[14] A. Tabata, A.P. Lima, L.K. Teles, L.M.R. Scolfaro, J.R. Leite, V. Lemos, B. Schöttker, T. Frey, D. Schikora, K. Lischka, Appl. Phys. Lett. 74, 362 (1999)

[15] M.E. Sherwin, T.J. Drummond, J. Appl. Phys. 69 8423 (1991).

[16] K. Kim, W.R.L. Lambrecht, B. Segall, Phys. Rev. B 53, 16310 (1996).

[17] A.F. Wright, J. Appl. Phys. 82, 2833 (1997).

[18] S. Saib, N. Bouarissa, Physica B 387, 377 (2007).

[19] S. Saib, N. Bouarissa, P. Rodriguez-Hernández, A. Muñoz, Comput. Mater. Sci. 81, 374 (2014).

[20] V.V. Sobolev, M.A. Zlobina, Semiconductors 33, 385 (1999).

[21] S.K. Pugh, D.J. Dugdale, S. Brand, R.A. Abram, Semicond. Sci. Technol. 14, 23 (1999).

[22] D. Vogel, P. Kruger, J. Pollmann, Phys. Rev. B 55 12836 (1997).

[23] N. Bouarissa, Eur. Phys. J. B 26, 153 (2002).

[24] J.R. Chelikowsky, Phys. Rev. B 35, 1174 (1987).

[25] W.B. Holzapfel, Rep. Prog. Phys. 59, 29 (1996) and references therein.

[26] S. Saib, N. Bouarissa, P. Rodriguez-Hernández, A. Muñoz, Physica B 403, 4059 (2008).

[27] A. Mujica, A. Rubio, A. Muñoz, R.J. Needs, Rev. Mod. Phys. 75, 863 (2003).

[28] N. Bouarissa, Mater. Chem. Phys. 73, 51 (2002).
[29] N. Bouarissa, Phys. B 406, 2583 (2011).

[30] C.S. Mishra, G. Palai, Optik 126, 4685 (2015).

[31] S. Daoud, N. Bioud, N. Bouarissa, Mater. Sci. Semicond. Process. 31, 124 (2015).

[32] K. Daviau, K.K.M. Lee, Crystals 8, 217 (2018).

[33] N. Bouarissa, K. Kassali, Phys. Status Solidi B 228, 663 (2001).

[34] S.J. Clark, M.D. Segall, C.J. Pickard, P.J. Hasnip, M.I.J. Probert, K. Refson, M.C. Payne, Z. Kristallogr. 220, 567 (2005).

[35] D. Vanderbilt, Phys. Rev. B 41, 7892 (1990).

[36] J.P. Perdew, A. Ruzsinszky, G.I. Csonka, O.A. Vydrow, G.E. Scuseria, L.A. Constantin, X. Zhou, K. Burke, Phys. Rev. Lett. 100, 136406 (2008).

[37] H.J. Monkhorst, J.D. Pack, Phys. Rev. B 13, 5188 (1976).

[38] V. Milman, M.C. Warren, J. Phys. Condens. Matter 13, 241 (2001).

[39] A. Reuss, Z. Angew, Math. Mech. 9, 49 (1929).

[40] W. Voigt, Lehrbuch der Kristallphysik, Teubner, Leipzig 1928 (in German).

[41] R. Hill, Proc. Phys. Soc. A 65, 349 (1952).

[42] R. Hill, J. Mech. Phys. Solids 11, 357 (1963).

[43] A. Khaldi, H. Ghodbane, N. Bouarissa, S. Daoud, L. Tabourot, J. Supercond. Nov. Magn. 30, 1533 (2017).

[44] A. Khaldi, N. Bouarissa, H. Ghodbane, L. Tabourot, Physica B 553, 6 (2019).

[45] L. Vocadlo, G.D. Price, I.G. Wood, Acta Crystallogr. $B$ 55, 484 (1999).

[46] S. Adachi, Properties of Group-IV, III-V and II-VI Semiconductors, Wiley, Chichester 2005.

[47] S. Adachi, in: Handbook on Physical Properties of Semiconductors, Vol. 2, III-V Compound Semiconductors, Kluwer Academic Publ., Boston (MA) 2004

[48] N. Bouarissa, Mater. Sci. Eng. B 100, 280 (2003).

[49] X.H. Deng, W. Lu, Y.M. Hu, H.S. Gu, Physica B 404, 1218 (2009)

[50] N. Bouarissa, Mater. Chem. Phys. 100, 41 (2006). 